\title{
Antibiotic susceptibility and imaging findings of the causative microorganisms responsible for acute urinary tract infection in children: a five-year single center study
}

Ji Eun Yoon, M.D., Wun Kon Kim, M.D., Jin Seok Lee, M.D. ${ }^{2}$, Kyeong-Seob Shin, M.D.', and Tae-Sun Ha, M.D.

Departments of Pediatrics ${ }^{1}$, Laboratory Medicine ${ }^{2}$, Chungbuk National University College of Medicine, Cheongju, Korea

Received: 10 March 2010, Revised: 8 June 2010

Accepted: 4 October 2010

Corresponding author: Tae Sun Ha, M.D.

Department of Pediatrics, Chungbuk National University College of Medicine, 410 Seongbong-ro, Heungdeok-gu,

Cheongju city, Chungbuk 361-763, Korea

Tel: +82.43-269-6374, Fax: +82.43-264-6620

E-mail: tsha@chungbuk.ac.kr

Copyright (C) 2011 by The Korean Pediatric Society
Purpose: We studied the differences in the antibiotic susceptibilities of the microorganisms that causeing urinary tract infections (UTI) in children to obtain useful information on appropriate drug selection for childhood UTI.

Methods: We retrospectively analyzed the antibiotic susceptibilities of 429 microorganisms isolated from 900 patients diagnosed with UTI in the Department of Pediatrics, Chungbuk National University Hospital, from 2003 to 2008.

Results: The most common causative microorganisms for UTI were Escherichia coli (81.4\%), Klebsiella pneumoniae (8.4\%), Enterobacter spp. (1.7\%), and Proteus spp. (0.4\%). E. coli showed relatively high susceptibility as compared to imipenem (100\%), amikacin $(97.7 \%)$, aztreonam $(97.9 \%)$, cefepime $(97.7 \%)$, and ceftriaxone $(97.1 \%)$, while it showed relatively low susceptibility to gentamicin (GM) (79.0\%), trimethoprim/sulfamethoxazole (TMP/SMX) $(68.7 \%)$, ampicillin/sulbactam (33.0\%), and ampicillin (AMP) (28.6\%). There were no significant differences in the image findings for causative microorganisms.

Conclusion: Gram-negative organisms showed high susceptibility to amikacin and third-generation cephalosporins, and low susceptibility to AMP, GM, and TMP/SMX. Therefore, the use of AMP or TMP/SMX as the first choice in empirical and prophylactic treatment of childhood UTI in Korea should be reconsidered and investigated further.

Keywords: Urinary tract infection, Escherichia coli, Antimicrobial susceptibility

This is an open-access article distributed under the terms of the Creative Commons Attribution Non-Commercial License (http://creativecommons.org/licenses/by$\mathrm{nc} / 3.0 /$ ) which permits unrestricted non-commercial use, distribution, and reproduction in any medium, provided the original work is properly cited. 


\section{Introduction}

Urinary tract infection (UTI) is the second most common infectious disease in toddlers ${ }^{1}$, and the most common disease in infants ${ }^{1-3)}$. UTI recurs easily if it is accompanied with anatomical anomalies of the urinary system. And, if it is not treated adequately or occurs recurrently, UTI may develop into chronic pyelonephritis, therefore, result in hypertension and loss of renal function, a condition normally seen in $15-20 \%$ of the cases of chronic renal failure ${ }^{1-4)}$.

In children, UTI frequency, clinical symptoms, and the causative pathogens, vary according to sex and age ${ }^{5,6)}$. Moreover, because of a wide variety of non-specific and systemic symptoms, it is difficult to perform tests for early diagnosis, and the resultant inaccurate diagnosis may lead to antibiotic abuse. Thus, in many cases, a severe renal injury occurs even before the UTI is diagnosed. For this reason, an early and accurate diagnosis through careful examination and tests can help in preventing severe renal injuries through adequate treatment and careful follow-up. Several studies on UTI pathogens and antibiotics have been conducted to determine the ideal approach for prompt treatment. However, the recent development of new antibiotics and the appearance and increase of antibioticresistant strains, which are created because of antibiotic abuse and inappropriate choice of antibiotics, have lead to changes in the antibiotic susceptibilities of the pathogens. This, in turn, influences the early choice of antibiotics ${ }^{7-9)}$.

Therefore, in the present study, we analyzed the findings for 900 pediatric patients diagnosed with childhood UTI at a university hospital and aimed to obtain information that could facilitate early selection of suitable antibiotics for childhood UTI.

\section{Materials and methods}

\section{Sulbjects}

We included 900 young inpatients and outpatients who presented to the Chungbuk National University Hospital between May 2003 and December 2008 with fever, urinary frequency, dysuria, vomiting, flank pain, and lower abdominal or suprapubic pain and were diagnosed with acute UTI. We performed urine culture tests and selected 429 samples in which urinary tract pathogens formed more than $1.0 \times 10^{5} \mathrm{CFU} / \mathrm{mL}$; we identified the causative pathogens from these 429 samples and determined the pathogen susceptibilities.

\section{Methods}

We performed urine tests on the basis of symptoms suggesting acute UTI, such as fever, urinary symptoms such as abnormal urinary frequency, abnormal urinary urgency, and dysuria, and abdominal symptoms such as flank, lower abdominal pain, and suprapubic pain. We identified 900 inpatients and outpatients who showed a white blood cell count of 6 or more per high-power field in urine samples under high-magnification microscopy and classified these patients into 2 groups: the acute pyelonephritis patient group and the acute cystitis patient group. For urine collection in boys, we turned up the foreskin and cleansed the urethral opening with a sponge dipped in $2 \%$ boric acid and then collected midstream urine in a sterilized plastic cup with a cap; for girls, we cleansed the genital region and the urethral opening with a sponge dipped in $2 \%$ boric acid and collected urine during urination. The collected urine samples were cultured, and for the 429 samples in which more than $1.0 \times 10^{5} \mathrm{CFU} /$ $\mathrm{mL}$ were formed, the UTI-causing pathogens were identified and antibiotic susceptibility was tested" ${ }^{9}$. In urine culture, we inoculated $0.001 \mathrm{~mL}$ of the collected urine into $5 \%$ sheep blood agar plate and MacConkey agar plate; the plates were cultured for a day at $35-36^{\circ} \mathrm{C}$ in an incubator, and the formed colonies were then identified using biochemical analysis and the Vitek system (bioMérienx, Durham, NC, USA). The antibiotic susceptibility test was performed using the Vitek GNS and GPS susceptibility card. The antibiotics used in the antibiotic susceptibility test were commonly used drugs in clinics. For gram-negative rods, we assessed the drug susceptibility to ampicillin, piperacillin, amikacin, gentamicin, tobramycin, cefazolin, cephalothin, sulperazone, ciprofloxacin, imipenem, and trimethoprim/sulfamethoxazole (TMP/SMX), etc., and for grampositive cocci, we assessed the drug susceptibility to penicillin, erythromycin, clindamycin, tetracyclin, ceftriaxone, cephalothin, ciprofloxacin, imipenem, and vancomycin, etc. Extended-spectrum $\beta$-lactamase (ESBL) production in Escherichia coli (E. coli) and Klebsiella pneumoniae (K. pneumoniae) was tested using the Vitek system or a double disk synergy test. In the double disk synergy test, an amoxicillin/clavulanic acid (AMC) disk was placed in the middle of Mueller-Hinton (MH) medium, and disks of aztreonam, cefotaxime, ceftazidime, and cefepime were placed around it at a distance of $25 \mathrm{~mm}$ from each other. A new inhibition zone between $\mathrm{AMC}$ and each disk or an increase in the size of the existing inhibition zones indicated the presence of ESBL-producing bacteria. For the 429 UTI patients, the distribution of pathogens according to the sex and age of the patient was investigated, the detection frequency of pathogens in each group was determined, and the pathogens' antibiotic susceptibility was retrospectively analyzed.

In this study, to examine urinary disorders that may accompany UTI, renal ultrasonography was performed first, and voiding cystourethrography (VCUG) was performed after the urine became aseptic. In addition, we performed ${ }^{99 \mathrm{~m}} \mathrm{Tc}$-dimercaptosuccinic acid (DMSA) renal scanning to find renal defects. 


\section{Statistical analysis}

Statistical analysis was performed using SPSS 12.0 (SPSS, Chicago, IL, USA). Frequency analysis and Chi-square test were performed and statistical significance was considered if $P<0.05$.

\section{Results}

1. Frequency of UTI according to the sex and age of the patients

With regard to the frequency of UTI according to sex and age, 257 (59.9\%) of the 429 young patients were male and 172 (40.1\%) were female; thus, the male-female ratio was 1.49:1. In addition, 301 patients (70.1\%) were aged less than 1 year, 57 (13.3\%) were aged 1-2 years, and 71 (16.6\%) were aged more than 2 years; thus, 358 (83.4\%) patients were aged 2 years or less.

With regard to the symptoms of UTI in the patients, 416 patients (96.9\%) had fever; 4 (0.9\%), flank pain; 3 (0.7\%), frequent urination; 2 (0.5\%), vomiting; 2 (0.5\%), dysuria; and $2(0.5 \%)$, lower abdominal pain (Table 1).

\section{Frequency of pathogens}

Only those strains that had a single colony count of over $1.0 \times 10^{5}$ CFU/mL were cultured, and among these, 413 (96.2\%) samples were of the following gram-negative strains: E.coli (349 samples; 81.4\%), Klebsiella spp. (36 samples; 8.4\%), Enterobacter spp. (7 samples; $1.7 \%)$, Proteus spp. (4 samples; $0.9 \%)$, Morganelle spp. (4 samples; 0.9\%), Pseudomonas spp. (3 samples; 0.7\%), Enterococcus spp. (9 samples; 2.1\%), and methicillin-resistant Staphylococcus aureus (MRSA) (3 samples; 0.7\%) (Table 2).

\section{Distribution of pathogens according to the sex and age of the patients}

Among the 349 patients with E. coli infection, 218 (62.5\%) were boys and 131 (37.5\%) were girls; among the 26 patients with Klebsiella spp. infection, 14 were boys and 22 were girls. Proteus spp. was isolated only from boys.

The distribution of pathogens according to the age was as follows:

Table 1. Age and Sex Distribution of 429 Children with Acute Urinary Tract Infections

\begin{tabular}{lccr}
\hline \multirow{2}{*}{ Age $(\mathrm{yr})$} & \multicolumn{2}{c}{$\operatorname{Sex}($ No., \%) } & \multirow{2}{*}{ Total (No., \%) } \\
\cline { 2 - 3 } & \multicolumn{1}{c}{ Male } & Female & \\
\hline$<1$ & $211(49.1)$ & $90(21.1)$ & $301(72.1)$ \\
1 & $26(6.0)$ & $31(7.2)$ & $57(13.3)$ \\
$>2$ & $20(4.7)$ & $51(11.9)$ & $71(16.6)$ \\
Total & $257(59.9)$ & $172(40.1)$ & $429(100)$ \\
\hline
\end{tabular}

E. coli was seen in both infants and toddlers, Klebsiella in infants, and Proteus and Morganelle in toddlers. In all the age groups, E. coli was the most frequently observed pathogen.

\section{Antibiotic susceptibilitities of different pathogens}

Among the strains cultured in the urine culture test, the antibiotic susceptibilities of Enterobacteriaceae were as follows: imipenem, 100\%; amikacin, 97.5\%; cefepime, 97.45\%; aztreonam, 96.27\%; ceftriaxone, 95.61\%; cefoxitin, 93.67\%; and ciprofloxacin, $93.02 \%$.

E. coli showed its highest susceptibility to imipenem (100\%), and the bacterium showed lower susceptibility to aztreonam (97.9\%), amikacin (97.7\%), cefepime (97.7\%), and ceftriaxone (97.1\%). In contrast, it showed low susceptibility to gentamicin (79\%), TMP/ SMX (68.7\%), ampicillin (23.6\%), and ampicillin/sulbactam (33.0\%) (Table 3).

\section{Change in the antibiotic susceptibility rates during 2 periods (2003-2005 and 2006-2008)}

For the strains cultured in the urine culture test, we compared the results of the antibiotic susceptibility tests in the 2 periods, namely, 2003-2005 and 2006-2008. We found that imipenem, amikacin, aztreonam, cefepime, ceftriaxone, and ciprofloxacin maintained a high antibiotic susceptibility of over $80 \%$ without significant change

Table 2. Microorganisms Isolated from 429 Episodes of Urinary Tract Infection

\begin{tabular}{lc}
\hline Microoganisms & No. of isolated (\%) \\
\hline Gram negative organisms & $349(81.4)$ \\
Escherichia coli & $33(7.7)$ \\
Klebsiella pneumoniae & $3(0.7)$ \\
Klebsiella oxytoca & $3(0.7)$ \\
Enterobacter aerogenes & $2(0.5)$ \\
Enterobacter asburiae & $2(0.5)$ \\
Enterobacter cloacae & $3(0.7)$ \\
Proteus mirabilis & $1(0.2)$ \\
Proteus penneri & $4(0.9)$ \\
Morganella morganii & $1(0.2)$ \\
Serratia marcescens & $1(0.2)$ \\
Citrobacter braakii & $5(1.2)$ \\
Citrobacter freundii complex & $3(0.7)$ \\
Pseudomonas aeruginosa & \\
Gram positive organisms & $3(0.7)$ \\
Staphylococcus aureus & $5(1.2)$ \\
Enterococcus avium - (Group D) & $4(0.9)$ \\
Enterococcus faecium - (Group D) & $429(100)$ \\
Total &
\end{tabular}


Table 3. Antibiotic Susceptibility of Isolated Microorganisms

\begin{tabular}{|c|c|c|c|c|c|c|c|c|c|c|c|c|c|c|}
\hline \multirow{2}{*}{ Microorganism (N) } & \multicolumn{14}{|c|}{ Antibiotic susceptibility\% } \\
\hline & IPM & AN & ATM & $\mathrm{CZ}$ & FEP & FOX & TZP & TOB & $\mathrm{AM}$ & GM & $\mathrm{CIP}$ & SXT & SAM & $\mathrm{CRO}$ \\
\hline E. coli (349) & 100 & 97.7 & 97.9 & 86.5 & 97.7 & 97.4 & 94.2 & 82.7 & 28.6 & 79.0 & 91.9 & 68.7 & 33.0 & 97.1 \\
\hline K. pneumoniae (33) & 100 & 93.9 & 90.9 & 69.6 & 93.9 & 78.7 & 84.8 & 87.8 & 3 & 87.8 & 100 & 93.9 & 52 & 92 \\
\hline K. oxytoca (3) & 100 & 100 & 100 & 0 & 100 & 100 & 100 & 100 & 0 & 100 & 100 & 100 & 50 & 100 \\
\hline E. aerogenes (3) & 100 & 100 & 100 & 28.5 & 100 & 0 & 85.7 & 100 & 0 & 100 & 100 & 85.7 & 20 & 100 \\
\hline E. cloacae (2) & 100 & 100 & 25 & 0 & 87.5 & 0 & 25 & 87.5 & 0 & 87.5 & 100 & 87.5 & 0 & 25 \\
\hline P. penneri (1) & 100 & 100 & 100 & 0 & 100 & 100 & 100 & 100 & 0 & 100 & 100 & 0 & 100 & 100 \\
\hline P. mirabilis (3) & 100 & 100 & 100 & 66.6 & 100 & 100 & 100 & 100 & 100 & 100 & 100 & 100 & 66.6 & 100 \\
\hline M. morganii (4) & 100 & 100 & 75 & 0 & 100 & 75 & 100 & 100 & 0 & 100 & 100 & 100 & 50 & 75 \\
\hline C. braakii (1) & 100 & 100 & 0 & 0 & 100 & 100 & 0 & 100 & 0 & 0 & 0 & 100 & 0 & 0 \\
\hline C. freundii complex (5) & 100 & 100 & 80 & 20 & 100 & 40 & 100 & 100 & 40 & 100 & 100 & 100 & 80 & 80 \\
\hline S. marcescens (1) & 100 & 100 & 50 & 0 & 100 & 50 & 100 & 50 & 0 & 50 & 50 & 100 & 50 & 50 \\
\hline Total (405) & 100 & 97.5 & 96.2 & 81.16 & 97.45 & 93.67 & 93.02 & 83.91 & 25.6 & 93.02 & 80.4 & 71.8 & 35.4 & 95.61 \\
\hline
\end{tabular}

Abbreviations: IPM, imipenem; AN, amikacin; ATM, aztreonam; CZ, cefazolin; FEP, cefepime; FOX, cefoxitin; TZP, peperacillin/tazobactam; TOB, tobramycin; AM, ampicillin; CIP, ciprofloxacin; GM, gentamicin; SXT, trimethoprim/sulfomethoxazol; SAM , ampicillin/sulbactam; CRO, ceftriaxone

Table 4. Comparison of Etiologic Organisms according to the Voiding Cystourethrography Findings

\begin{tabular}{lccccccc}
\hline \multirow{2}{*}{ Microorganisms } & \multicolumn{7}{c}{ VCUG Findings (No., \%) } \\
\cline { 2 - 7 } & Normal & GI & G II & G III & G IV & G V \\
\hline E. coli & $234(84.5)$ & $4(1.4)$ & $8(2.9)$ & $16(5.8)$ & $6(2.2)$ & $9(3.2)$ & Total \\
K. pneumoniae & $11(61.1)$ & $1(5.6)$ & $1(5.6)$ & $1(5.6)$ & $2(11.1)$ & $2(11.1)$ & $18(100)$ \\
\hline
\end{tabular}

Abbreviation: G, Grade ; VCUG, Voiding cystourethrography

in both the periods $(P>0.05)$. In contrast, gentamicin, TMP/SMX, ampicillin/sulbactam, and ampicillin showed consistently low susceptibilities of 50-80\% without significant differences between the 2 periods ( $P>0.05$, data not shown as a table).

\section{ESBL isolation rate}

Among the 429 strains cultured in the urine culture test, 17 (4.0 \%) were ESBL-positive; this isolation rate was much lower than that reported among adult patients (over 10\%, unpublished data). According to a recent investigation by our hospital, ESBL was isolated in $16 \%$ of $K$. pneumoniae infections and $10 \%$ of $E$. coli infections reported in 2003 and $24.7 \%$ and $10 \%$, respectively, of the cases reported in 2005.

\section{Radiological examination}

Among the 429 young patients diagnosed with UTI, 334 (77.9\%) underwent renal ultrasonography and 315 (73.4\%) underwent VCUG. Hydronephrosis was detected in 108 cases (32.3\%), and renal agenesis, double ureter, etc. were detected in 25 cases (7.5\%). Vesicoureteral reflux observed through VCUG was Grade I in 5 patients (1.6\%), Grade II in 10 patients (3.1\%), Grade III in 17
Table 5. Comparison of Etiologic Organisms according to the Kidney Ultrasonography Findings

\begin{tabular}{lcccc}
\hline \multirow{2}{*}{ Microorganisms } & \multicolumn{3}{c}{ Kidney Ultrasonography (No., \%) } \\
\cline { 2 - 4 } & Normal & Hydronephrosis & Others & \multicolumn{1}{c}{ Total } \\
\hline E. coli & $171(59.8)$ & $93(32.5)$ & $22(7.7)$ & $286(100)$ \\
K. pneumonia & $12(52.2)$ & $8(34.8)$ & $3(13.0)$ & $23(100)$ \\
\hline
\end{tabular}

patients (5.4\%), and Grade IV-V in 21 patients (6.7\%). Overall, vesicoureteral reflux was detected in 53 (16.8\%) out of the 315 patients who underwent VCUG. The results of radiological examinations of the UTIs caused by $E$. coli and $K$. pneumoniae were compared, but no significant differences were observed $(P>0.05)$ (Table 4, 5).

\section{8. ${ }^{99 \mathrm{~m}}$ TC-DMSA renal scanning}

Among the 429 young patients diagnosed with UTI, 340 (79.3\%) underwent ${ }^{99 \mathrm{~m}} \mathrm{Tc}-\mathrm{DMSA}$ renal scanning; among these, 167 patients (49.1\%) had a renal scar on 1 side and 40 (11.8\%) had renal scars on both sides. Renal defects were found in 207 (60.9\%) out of the 340 patients that had undergone ${ }^{99 \mathrm{~m}} \mathrm{Tc}-\mathrm{DMSA}$ renal scan. The results of ${ }^{99 \mathrm{~m}}$ Tc-DMSA renal scans of patients with $E$. coli UTI and $K$. 
Table 6. Comparison of Etiologic Organisms According to the ${ }^{99 \mathrm{~m}} \mathrm{Tc}$ DMSA Renal Scan Findings

\begin{tabular}{lrrrr}
\hline \multirow{2}{*}{ Microorganisms } & \multicolumn{4}{c}{${ }^{99 m}$ Tc-DMSA renal scan (No., \%) } \\
\cline { 2 - 5 } & Normal & Unilateral & Bilateral & \multicolumn{1}{c}{ Total } \\
\hline E. coli & $126(40.5)$ & $152(48.1)$ & $33(10.6)$ & $311(100)$ \\
K. pneumoniae & $7(33.3)$ & $11(52.4)$ & $3(14.3)$ & $21(100)$ \\
\hline
\end{tabular}

pneumoniae UTI were compared, but no significant difference was observed $(P>0.05)$ (Table 6).

\section{Discussion}

UTI is one of the most common bacterial diseases in infants and children; however, $1-3 \%$ of the cases are asymptomatic, and infants and toddlers usually show non-specific symptoms such as fever. Therefore, early detection of this disease is difficult without a proper understanding of the characteristics of UTI; if the infection is not diagnosed early and treated with a suitable antibiotic, it may result in serious renal damage ${ }^{1-3)}$. For example, if UTI is misdiagnosed as simple fever, upper respiratory tract infection, or enteritis, then its symptoms may temporarily ease through short-term administration of an antipyretic or antibiotic, but improper treatment and inability to recognize an accompanying anomaly of the urine system may cause the recurrence of UTI. This may eventually cause a severe renal injury and renal insufficiency and result in serious sequelae such as renal hypertension and chronic renal failure ${ }^{4-7,10)}$.

The frequency of UTI in children varies according to age and sex. According to reports ${ }^{11-14)}$, in foreign countries, symptomatic bacteriuria is observed in $2.3-3.6 \%$ of male neonates, $0.3-0.9 \%$ of female neonates, $0.2-0.4 \%$ of male infants and toddlers, $0.7-2.6 \%$ of female infants and toddlers, $0.04-0.2 \%$ of school-age boys, and $1.2-1.9 \%$ of school-age girls. In addition, UTI is observed in 3-5\% of female children and around $1 \%$ of male children ${ }^{11-14)}$. In our study, among the 429 patients hospitalized for UTIs, 257 patients (59.9\%) were boys and 172 (40.1\%) were girls (male-female ratio, 1.49:1), and the percentage of patients younger than 1 year was somewhat higher than those in previous Korean reports ${ }^{15-17)}$.

Although radiological examinations are usually performed to detect structural abnormalities in the urinary system of children with $\mathrm{UTI}^{1,2)}$, in principle, renal ultrasonography should be performed for every case of childhood UTI regardless of its stage. In this study, 334 (77.9\%) out of the 429 patients diagnosed with UTI underwent renal ultrasonography and 133 (39.8\%) of these patients had normal results in renal ultrasonography. This rate is somewhat higher than the values in Korean reports, such as the 30.7\% (42/137) reported by Kwon et al. $^{18)}$ and the $10.3 \%(7 / 68)$ reported by Lee et al. ${ }^{19)}$. The difference in the rate of detection of anomalies may be attributed to the advances in imaging machines and anomaly classification.

In this study, VCUG was performed for 315 cases (73.4\%) and vesicoureteral reflux was observed in 53 cases (16.8\%). This frequency of vesicoureteral reflux is lower than that seen in other Korean reports $(18-50 \%)^{18-21)}$, but this difference may be because we included fewer cases of UTIs such as urethritis and cystitis. Among the 340 cases that underwent ${ }^{99 \mathrm{~m}}$ Tc-DMSA renal scanning, 207 cases $(60.9 \%)$ showed renal scars, which is somewhat higher than the number in previous Korean reports ${ }^{18,22)}$.

Most of the pathogens causing UTI in children were $E$. coli, and $10-15 \%$ of the infections were caused by Klebsiella spp., Enterobacter spp., Proteus spp., Pseudomonas, etc ${ }^{13-15,23-29)}$. According to Cohen et $\mathrm{al}^{25)}$, UTI caused by $E$. coli was most frequently seen among boys, but the frequency of UTI caused by $E$. coli was significantly different between boys and girls, that is, it was $88 \%$ in girls but $62 \%$ in boys. $E$. coli infections accounted for $81.4 \%$ of the UTIs included in this study, and this value was similar to the values in other reports ${ }^{13-15,23-28)}$.

The antibiotic susceptibility of E. coli, which was the most common causative pathogen of UTI in children during the period of this study, was highest to imipenem (100\%) followed by aztreonam (97.9\%), amikacin (97.7\%), cefepime (97.7\%), and ceftriaxone (97.1\%). Thus, E.coli still showed susceptibility to old-generation cephalosporins and some aminoglycosides, but it showed low susceptiblity to ampicillin (23.6\%), ampicillin/sulbactam (33.0\%), etc., which raises a question on the effectiveness of these drugs for treating UTI in children. According to other previous Korean studies on susceptibility, Yang et al. ${ }^{27)}$ reported that the susceptibility of E.coli was the highest to moxalactam (100\%) followed by amikacin (96.9\%), tobramycin (94.3\%), gentamicin (89.6\%), cefamandole (62.5\%), and TMP-SMX (41.7\%), and the pathogen showed very low susceptibility to ampicillin (9.0\%). In the report by Cha et al. ${ }^{28)}$,E.coli showed susceptibility to cefotaxime $(91.4 \%)$, cefamandole (88.1\%), amikacin (84.0\%), ampicillin/sulbactam (66.7\%), and gentamicin (61.7\%), and in the report by Kang et al. ${ }^{29)}$ it showed susceptibility to ceftriaxone (95.9\%), amikacin (95.8\%), ceftazidime (91.8\%), cefazolin (85.7\%), tobramycin (62.7\%), gentamicin (58.7\%), TMP-SMX (42.3\%), ampicillin/sulbactam (42.2\%), and ampicillin (16.9\%). These results show a similar tendency as that observed in our report.

In our study, Enterobacteriaceae showed high susceptibility to imipenem (100\%), amikacin (97.5\%), cefepime (97.45\%), aztreonam (96.27\%), ceftriaxone (95.61\%), cefoxitin (93.67\%), ciprofloxacin (93.02\%), tobramycin (83.91\%), cefazolin $(81.16 \%)$, gentamicin (80.4\%), etc., and low susceptibility to ampicillin/sulbactam (35.4\%), ampicillin (25.6\%), etc. This finding suggests that Enterobacteriaceae 
have natural resistance to ampicillin in Korean population.

Infection with ESBL-producing strains is usually a hospital-acquired infection occurring in intensive care units (ICU), and its frequency has been increasing rapidly during the last 10 years, although the frequency of this infection varies across regions. In the U.S., $1-8 \%$ of $E$. coli and $7-34 \%$ of $K$. pneumoniae strains isolated from patients with hospitalacquired infection were ESBL-positive ${ }^{29)}$. Further, in Korea, ESBL production was reported in $4.8-13.9 \%$ of $E$. coli and $22.5-25.6 \%$ of K. pneumoniae infections. Among young children, ESBL-positive infections were mainly seen in neonates who were under intensive care and had weak immune function, while some previous studies in Korea reported sepsis and UTI in pediatric and neonatal ICU patients. However, community-acquired infection has been reported rarely, with some reports of occurrence in nursing home residents with a history of antibiotic medication and hospitalization ${ }^{30,31)}$. ESBLs are enzymes that hydrolyze oxyimino- $\beta$-lactam antibiotics by a plasmid-mediated mechanism and induce resistance to oxyimino-cephalosporins and aztreonam; these enzymes are inhibited by a $\beta$-lactamase inhibitor, clavulanic acid ${ }^{32,33)}$.

In this study, most of the patients from whom ESBL-producing $E$. coli were isolated had presented to the hospital with fever of unknown origin and were diagnosed with the first febrile UTI. Therefore, their infection was obviously community-acquired rather than hospitalacquired. Among the 429 strains cultured in the urine culture test, 17 (4.0\%) were ESBL-positive, and the isolation rate of ESBL-positive strains was much lower than that among adult patients investigated in this hospital (data not shown).

In conclusion, according to the antibiotic-susceptibility data reported by Korean studies including this study, pathogens causing UTIs show susceptibility to antibiotics such as amikacin and cephalosporins; however, they show low susceptibility to ampicillin/ amoxicillin and most of the aminoglycosides. Ampicillin and gentamicin are used as early empirical remedies, and TMP-SMX is recommended as a prophylactic antibiotic for the treatment of UTIs in children. However, according to the results of our study, the resistance rates for these antibiotics are relatively high; therefore, clinicians should re-evaluate the use of these antibiotics as early empirical and prophylactic antibiotics for UTI cases in children in Korea.

\section{Acknowledgement}

This work was supported by the research grant of the Chungbuk National University in 2009.

\section{References}

1) Elder JS. Urinary tract infections. In: Kliegman RM, Behrman RE, Jensen HB, Stanton BF, editors. Nelson Textbook of Pediatrics. 18th ed. Philadelphia: WB Saunders, 2007:2223-8.

2) Wald E. Urinary tract infections in infants and children. Curr Opin Pediatr 2004;16:85-8.

3) Bloomfield P, Hodson EM, Craig JC. Antibiotics for acute pyelonephritis in children. Cochrane Database Syst Rev 2005:CD003772.

4) Choi SK. Urinary tract infection and its diagnosis in children. J Korean Pediatr Soc 1987;30:945-52.

5) Bergstrom T, Larson H, Lincoln K, Winberg J. Studies of urinary tract infections in infancy and childhood. J Pediatr 1972;80:858-66.

6) Bergstrom T. Sex differences in childhood urinary tract infection. Arch Dis Child 1972;47:227-32.

7) Sidor TA, Resnick MI. Urinary tract infection in children. Pediatr Clin North Am 1983;30:323-32.

8) Erb A, Sturmer T, Marre R, Brenner H. Prevalence of antibiotic resistance in Escherichia coli: overview of geographical, temporal, and methodological variations. Eur J Clin Microbiol infect Dis 2007;26:83-90.

9) Gupta K, Hooton TM, Stamm WE. Increasing antimicrobial resistance and the management of uncomplicated community-acquired urinary tract infections. Ann Intern Med 2001;135:41-50.

10) Hanson LA, Ahlstedt S, Jodal U, Kaijser P, Larsson G, Lindin-Janson G, et al. The host-parasite relationship in urinary tract infections. Kidney Int Suppl 1975;4: S28-34.

11) Maherzi M, Guignard JP, Torrado A. Urinary tract infection in high-risk newborn infants. Pediatrics 1978;62:521-3.

12) Mair MI. High incidence of asymptomatic urinary tract infection in infant and schoolgirls. Scott Med J 1973;18:51-5.

13) Khan AJ, Ubriani RS, Bombach E, Agbayani MM, Ratner H, Evans HE. Initial urinary tract infection caused by Proteus mirabilis in infancy and childhood. J Pediatr 1978;93:791-3.

14) Winberg J, Andersen HJ, Bergström T, Jacobsson B, Larson H, Lincoln K. Epidemiology of symptomatic urinary tract infection in childhood. Acta Paediatr Scand Suppl 1974;252:1-20.

15) Cha YH, Choi HR, Jang SH, Ahn YM. A clinical study on urinary tract infection in infants and children. J Korean Pediatr Soc 1994;37:1488-99.

16) Kang YD, Kim NS, Oh SH. Causative organisms of urinary tract infection in children and their antibiotic susceptibility. Korean J Pediatr 2004;47:1065-71.

17) Lee SH, Yang SJ, Shin JH, Lee WG. Sex and age differences of etiologic organisms in urinary tract infection. J Korean Pediatr Soc 1987;30:11006.

18) Kwon YD, Oh SH. Antibiotic sensitivity pattern of pathogens from children with UTI. J Korean Soc Pediatr Nephrol 2006;10:182-91.

19) Lee YK, Lee HC, Chun JM, Yoon SY, Lee WG, Shin SM. Antibiotic sensitivity to the major causative organisms of acute urinary tract infection in children. Korean J Pediatr 2005;48:760-5.

20) Yoe MH, Kim WY, Kim JS, Lee SG, Kang IJ. Comparison of the 3-imaging procedures(IVP, VCUG \& USG) on children with urinary tract infection. J Korean Pediatr Soc 1990;33:332-40.

21) Lee HD, Kim CY. A clinical study of infants and children with urinary tract infection. J Korean Pediatr Soc 1989;32:1706-14. 
22) Lee $\mathrm{SH}$, Noh SH, Oh JE, Kim MS, Lee DY. Predictive Value for Vesicoureteral Reflux in Children with Urinary Tract Infection. J Korean Soc Pediatr Nephrol 2008;12:62-9.

23) Hallett RJ, Pead L, Maskell R. Urinary tract infection in boys. Lancet 1976;2:1107-10.

24) Hoberman A, Chao HP, Keller DM, Hickey R, Davis HW, Ellis D. Prevalence of urinary tract infection in febrile infants. J Pediatr 1993; 123:17-23.

25) Cohen M. The first urinary tract infection in male children. Am J Dis Child 1976;130:810-3.

26) Chi HM, Kwahk JH, Lee JH, Park HW. Incidence of Escherichia coli and its susceptibility to antimicrobials in childhood urinary tract infection. J Korean Soc Pediatr Nephrol 2006;10:18-26.

27) Yang KS, Ahn MS, Kim HB, Oh JS. Clinical studies of urinary tract infection in infant and children. J Korean Pediatr Soc 1989;32:533-41.
28) Kang YD, Kim NS, Oh SH. Causative organisms of urinary tract infection in children and their antibiotic susceptibility. Korean J Pediatr 2004; $47: 1065-71$.

29) Livermore DM. $\beta$-Lactamases in laboratory and clinical resistance. Clin Microbiol Rev 1995;8:557-84.

30) Lee JW, Shin JS, Seo JW, Lee MA, Lee SJ. Incidence and risk factors for extended-spectrum $\beta$-lactamase-producing Escherichia coli in community-acquired childhood urinary tract infection. J Korean Soc Pediatr Nephrol 2004;8:214-22.

31) Jacoby GA, Medeiros AA. More extended-spectrum $\beta$-lactamases. Antimicrob Agents Chemother 1991;35:1697-704.

32) Bush K, Jacoby GA, Medeiros AA. A functional classification scheme for $\beta$-lactamases and its correlation with molecular structure. Antimicrob Agents Chemother 1995;39:1211-33.

33) Pai $H$. The characteristics of extended-spectrum $\beta$-lactamases in Korean isolates of Enterobacteriaceae. Yonsei Med J 1998;39:514-9. 